\title{
Propriétés des argiles marines de grande profondeur ${ }^{(1)}$
}

\section{J.-L. FAVRE}

Laboratoire de mécanique des sols structures

et matériaux

UMR CNRS 8579

École centrale Paris grande voie des Vignes 92295 Châtenay-Malabry

Cedex

jean-louis.favre@ecp.fr

\section{HATTAB}

Laboratoire de physique et mécanique des matériaux ISGMP-UMR CNRS 7554 fle du Saulcy 57045 Metz Cedex 1 hattab@univ-metz.fr
Quatorze mètres d'une carotte d'argile marine de grande profondeur, provenant du golfe de Guinée, sont analysés suivant un pas très fin de $16,7 \mathrm{~cm}$. Ces propriétés sont comparées, d'une part, au comportement de référence des argiles minérales remaniées normalement consolidées établi par Biarez et Favre (1975, 1977), et, d'autre part, à la courbe de compression intrinsèque (ICL) proposée par Burland (1990). Les essais portent sur les paramètres d'identification $w_{L}, w_{p}, V B S, w_{n a t}$ et sur les paramètres mécaniques $c_{\mathrm{u}}, \mathrm{C}_{\mathrm{c}}, \mathrm{C}_{v}, \sigma_{c}^{\prime}, \mathrm{OCR}$. La finesse du pas ainsi que la concordance des profils, permettent de mettre en évidence une genèse complexe ainsi que les spécificités de cette smectite marine naturelle : indice des vides de sédimentation, sensibilité, " surconsolidation ") apparente, celle-ci traduisant une cohésion de type physico-chimique.

Mots-clés : sédiments marins de grande profondeur, compressibilité, essais œdométriques, paramètres d'identification, cohésion non drainée.

\section{Properties of deepwater marine clays}

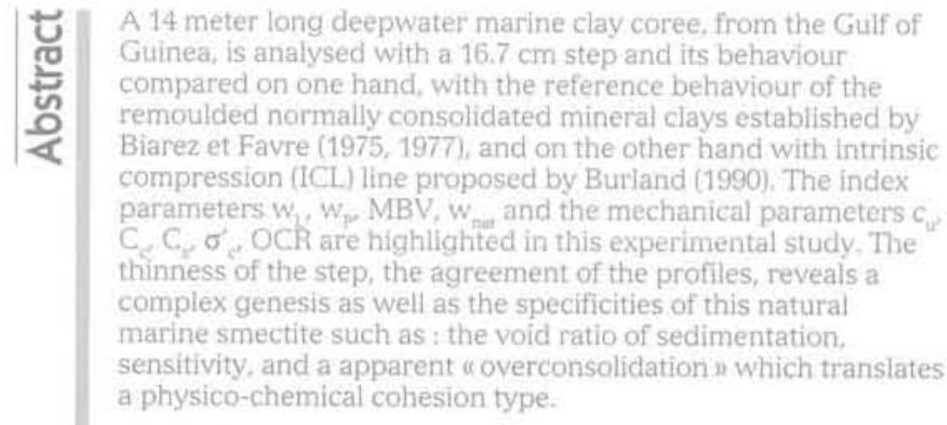

Key words : deepwater sediments, index properties, oedometric tests, compressibility, undrained cohesion.

\footnotetext{
(1) Étude dans le cadre d'un contrat CLAROM a Sols marins grande profondeur $\mathrm{p}$, sur une carotte Stacor provenant du site B du golfe de Guinée. Les partenaires du projet étant: IFP, Ifremer. Fugro-France, Saipem-SA, Technip et Total.
} 


\section{Introduction}

Les paramètres d'identification liés à la nature minéralogique des particules, et représentés par les limites d'Atterberg, et les paramètres physiques qui traduisent l'arrangement spatial des particules, permettent de résumer les propriétés des sédiments argileux. Les particules peuvent être liées entre elles par une cimentation pouvant présenter un grand nombre d'aspects et qui apparaît comme un paramètre supplémentaire important du comportement phénoménologique (Biarez et al., 1998; Ismail et al., 2002). Lorsque la liaison entre les particules n'existe pas ou qu'elle a été détruite, le sol devient remanié. Il est courant, pour l'étude du comportement mécanique, de reconstituer le matériau au laboratoire dans les conditions normalement consolidées ou surconsolidées (Biarez et Hicher, 1994; Hattab et Hicher 2004). Entre autres, Lambe et Martin (1957), Cozzolino (1961), et Favre (1972) ont proposé et rassemblé dans la littérature un grand nombre de corrélations entre les propriétés mécaniques des argiles remaniées et leurs paramètres physiques et d'identification. Beaucoup sont parues depuis 1971 dans les comptes rendus des « Conférences internationales sur les Applications des statistiques et probabilités en génie civil », ou ont été rassemblées par des auteurs comme Favre (1980) et Magnan (1982).

L'étude des sédiments marins a été abordée par plusieurs auteurs qui ont mis en évidence leurs spécificités. Les propriétés physiques et mécaniques peuvent se différencier en fonction de la provenance du sol marin (terrigène ou pélagique), néanmoins dans leur grande majorité, ces sédiments ont en surface des teneurs en eau élevées, une grande compressibilité et une très faible résistance au cisaillement. Ce sont des sols très hétérogènes qui peuvent évoluer, y compris dans les cas de sols normalement consolidés, d'une manière significative en fonction de la profondeur. La présence marquée d'éléments carbonatés en surface, mais aussi d'autres facteurs, pourrait expliquer la cimentation souvent observée à ces profondeurs, sous forme d'une (" surconsolidation ») apparente (Yin, 1999; Ohtsubo et al., 2000). Biarez et al. (1977) supposent ainsi que la résistance au cisaillement non drainé $\mathrm{c}_{\text {u }}$ est fonction de trois paramètres, l'angle de frottement interne du matériau $\left(\phi^{\prime}\right)$, la minéralogie $\left(\mathrm{I}_{\mathrm{p}}\right)$ et la cimentation. Des corré-

\section{NOTATIONS}

$W_{\text {nat }}$ : teneur en eau en place

$w_{1}, w_{p}$ : limites de liquidité, limite de plasticité

$I_{L}, I_{P}$ : indice de liquidité, indice de plasticité

$\mathrm{e}^{-} \quad$ indice des vides

$e_{1}, e_{p}$ : indice des vides correspondants aux limites

$\gamma \quad$ : poids spécifique déjaugé en place

$\sigma_{y}^{\prime} \quad$ : contrainte verticale en place

(correspondant aux poids des terres)

$\sigma_{c} \quad$ : contrainte de consolidation de terrain

$c_{\text {. }}$ : cohésion non drainée

VBS : valeur au bleu de Méthylène

OCR : degré de surconsolidation

$\mathrm{C}_{\mathrm{c}} \mathrm{C}_{\mathrm{s}}$ : indice de compression et indice de gonflement

$\mathrm{N}_{\mathrm{k}}: \mathrm{N}_{\mathrm{k}}=\frac{\mathrm{c}_{\mathrm{u}}}{\mathrm{q}_{\mathrm{c}}}$ lations à des échelles différentes ont été établies pour les argiles marines, et nous pouvons citer, par exemple comme études récentes, entre autres celles de Ohtsubo et al. (2000) qui montre, à partir de deux types d'argile, celle de Bangkok et celle d'Ariak, l'influence de la minéralogie et des propriétés chimiques des sédiments sur les paramètres géotechniques d'identification. Yin (1999) sur les sédiments marins de Hong Kong montre la corrélation entre le pourcentage d'argile contenu dans les sédiments et les caractéristiques mécaniques de compressibilité, de rupture ainsi que d'identification.

La structure du sédiment mise en place lors de la sédimentation a donc une influence certaine très importante sur le comportement mécanique, en l'occurrence sur la compressibilité (Burland, 1990; Cotecchia et Chandler, 1997). Néanmoins le comportement mécanique de la même argile, mais qui a été remaniée et reconstituée au laboratoire, peut constituer un repère fixe servant d'asymptote au comportement des mêmes argiles mais non remaniées. Dans ce cadre et pour cette étude, il nous a paru intéressant de situer l'argile marine grande profondeur, provenant du golfe de Guinée (GoG), parmi les corrélations établies par Biarez et Favre $(1975,1977)$, en utilisant un cadre logique pour celles-ci afin d'expliquer ses particularités par rapport aux argiles remaniées.

\section{2}

\section{Comportement de référence des argiles remaniées normalement consolidées}

Biarez et Favre $(1975,1977)$ ont établi un cadre logique pour établir ce comportement : le sol, milieu granulaire discontinu (MD), est idéalisé en un milieu continu fictif (MC). Ce dernier apparaît comme l'intégration des propriétés des grains (appelées « nature des grains $n$ ), dans leur configuration spatiale (appelée « arrangement des grains $\eta)$, et mécanique aux limites (dont le confinement peut rendre compte). Ainsi ils ont proposé la logique suivante pour les liaisons statistiques :

\begin{tabular}{|l|l|}
$\begin{array}{l}\text { Nature des } \\
\text { grains (MD) }\end{array}$ & $+\begin{array}{l}\text { Arrangement et } \\
\text { confinement des grains } \\
\text { (MD) }\end{array}$
\end{tabular}$\Rightarrow$\begin{tabular}{l}
$\begin{array}{l}\text { Propriétés } \\
\text { mécaniques du sol } \\
\text { (MC) }\end{array}$ \\
\hline
\end{tabular}

A travers une très abondante bibliographie, Favre (1972, 1980) a montré, pour les argiles remaniées, que les paramètres de « nature des grains » les plus explicatifs étaient les limites d'Atterberg, elles-mêmes très liées entre elles pour les argiles minérales (relation (1) du tableau 1), d'où le modèle proposé dans la figure 1. De même, il a montré qu'un chargement œdométrique de $6,5 \mathrm{kPa}$ donne un indice des vides correspondant à $\mathrm{w}_{1}$ et qu'un chargement de $1 \mathrm{MPa}$ donne un indice des vides correspondant à $w_{\mathrm{p}}$ (relation (2) du tableau I et figure 2). Ceci conduit à l'expression de $C_{\text {de l'équa- }}$ tion (3) ou (6) et à l'équation (4) en portant (1) dans (3). Skempton ayant déjà proposé dès 1944 pour les argiles remaniées la relation (5) avec laquelle on obtient une sous-estimation de $C_{c}$ de $10 \%$ pour $w_{L}=40$ et de $5 \%$ pour $w_{L}=70$. Maís vu les incertitudes sur les contraintes de consolidation et sur la corrélation de Skempton, Favre et al. (2002) ont conservé le modèle simple (2) et (4) pour l'essai œdométrique. On obtient ainsi un abaque unique, pour toutes les argiles de limite 
de liquidité $w_{L}$ comprise entre 20 et $160 \%$ avec $\sigma_{v}$ en $\mathrm{kPa},(7)$. Ainsi l'arrangement apparait comme directement lié à la contrainte de consolidation à travers la minéralogie. Enfin, Favre (1980) avait aussi proposé la relation (8) indiquant le rapport $\mathrm{C} / \mathrm{C}$. Skempton (1954) a donné la corrélation (9) pour le calcul de $c_{u}$, où $\sigma_{c}$ est la contrainte de consolidation de terrain, c'est-à-dire, pour les sols normalement consolidés, le poids des terres, $\sigma_{\mathrm{y}}^{\prime}=\int \gamma$.dz, le poids spécifique des grains étant pris égal à $27 \mathrm{kN} / \mathrm{m}^{3}$. Biarez et Favre (1975) ont conservé cette corrélation (Fig. 3) qui permet d'avoir les valeurs du tableau Il en fonction seulement de $w_{t}$. Elle permet aussi, par l'intermédiaire de la contrainte effective verticale en place d'avoir une liaison statistique biunivoque entre l'indice des vides (ou l'indice de liquidité) des argiles minérales remaniées, normalement consolidées et leur cohésion non drainée ainsi que leur déformabilité à travers le paramétrage de $w_{\mathrm{L}}$ (Tableau III).

TABLEAUT Liaison entre les paramètres : corrélations. Correlations between parameters.

\begin{tabular}{|c|c|c|c|}
\hline $\begin{array}{c}I_{D}=0,73\left(w_{f}-13\right) \\
(\text { en } \%)\end{array}$ & (1) & $\begin{array}{c}\mathrm{Cc}=\frac{1_{p}}{81} \\
\left(I_{p} \text { en } \%\right)\end{array}$ & (6) \\
\hline $\begin{array}{l}\mathrm{w}_{\text {sat }}=\mathrm{w}_{1} \text { pour } \sigma_{y}-6,5 \mathrm{kPa} \\
\mathrm{w}_{\text {sat }}=\mathrm{w}_{p} \text { pour } \sigma_{v}-1 \mathrm{MPa}\end{array}$ & (2) & $I_{L}=0,46\left(3-\log \sigma_{\nu}^{\prime}\right)$ & (7) \\
\hline $\begin{array}{c}C_{t}=\frac{\left(w_{L}-w_{p}\right) 2.7 / 100}{\log (1.000 / 6,5)} \\
\left(w_{1} \text { et } w_{p} \text { en } \%\right)\end{array}$ & (3) & $\frac{C_{c}}{C s}=4$ à 6 & (8) \\
\hline $\begin{aligned} C_{c}= & 0,009\left(w_{L}-13\right) \\
& \left(w_{L} \text { en } \%\right)\end{aligned}$ & (4) & $C_{\mathrm{u}}=\left(0,11+0,0037 \mathrm{I}_{\mathrm{p}}\right) \sigma_{\mathrm{c}}$ & (9) \\
\hline $\begin{aligned} C_{c}= & 0,007\left(w_{2}-10\right) \\
& \left(w_{1} \text { en } \%\right)\end{aligned}$ & (5) & & \\
\hline
\end{tabular}

TAateau II: Paramètres mécaniques indépendants de l'arrangement : argiles.

Mechanical parameters independent of the arrangement: clays.

\begin{tabular}{c|c|c|c|c|c|c|c|c|c|c|c}
\hline $\mathrm{w}_{\mathrm{f}}$ & 20 & 30 & 40 & 50 & 60 & 70 & 80 & 90 & 100 & 150 & 200 \\
\hline $\mathrm{w}_{\mathrm{p}}$ & 15 & 18 & 20 & 23 & 26 & 28 & 31 & 34 & 37 & 50 & 64 \\
\hline $\mathrm{I}_{\mathrm{p}}$ & 5 & 12 & 20 & 27 & 34 & 42 & 49 & 56 & 63 & 100 & 136 \\
\hline $\mathrm{C}_{\epsilon}$ & 0,06 & 0,15 & 0,24 & 0,33 & 0,42 & 0,51 & 0,60 & 0,69 & 0,78 & 1,23 & 1,68 \\
\hline $\mathrm{c}_{\mathrm{u}} / \sigma_{c}^{\prime}$ & 0,13 & 0,15 & 0,18 & 0,21 & 0,24 & 0,27 & 0,29 & 0,32 & 0,34 & 0,48 & 0,61
\end{tabular}

TABLEAUIIIII Paramètres mécaniques dépendant de l'arrangement : argiles.

Mechanical parameters dependent of the arrangement : clays.

\begin{tabular}{c|c|c|c|c|c|c}
\hline $\mathrm{I}_{\mathrm{c}}$ & 0 & 0,25 & 0,50 & 0,75 & 1 & 1,25 \\
\hline $\mathrm{I}_{\mathrm{L}}$ & 1 & 0,75 & 0,50 & 0,25 & 0 & $-0,25$ \\
\hline $\begin{array}{c}\mathrm{c}_{\mathrm{u}}\left(\mathrm{w}_{\mathrm{L}}=50\right) \\
(\mathrm{kPa})\end{array}$ & 1,5 & 5 & 15 & 40 & 100 & 300 \\
\hline $\begin{array}{c}\mathrm{c}_{\mathrm{u}}\left(\mathrm{w}_{1}=100\right) \\
(\mathrm{kPa})\end{array}$ & 2,3 & 8 & 25 & 70 & 170 & 500 \\
\hline $\begin{array}{c}\mathrm{E}_{\text {oeedo }}\left(\mathrm{w}_{\mathrm{L}}=50\right) \\
(\mathrm{kPa})\end{array}$ & 90 & 300 & 1000 & 3400 & 11000 & 35000 \\
\hline $\begin{array}{c}\mathrm{E}_{\text {octodo }}\left(\mathrm{w}_{\mathrm{L}}=100\right) \\
(\mathrm{kPa})\end{array}$ & 62 & 200 & 600 & 1900 & 5700 & 16000 \\
\hline $\begin{array}{c}\sigma \\
(\mathrm{kPa})\end{array}$ & 7 & 23 & 90 & 300 & 1000 & 3500 \\
\hline
\end{tabular}

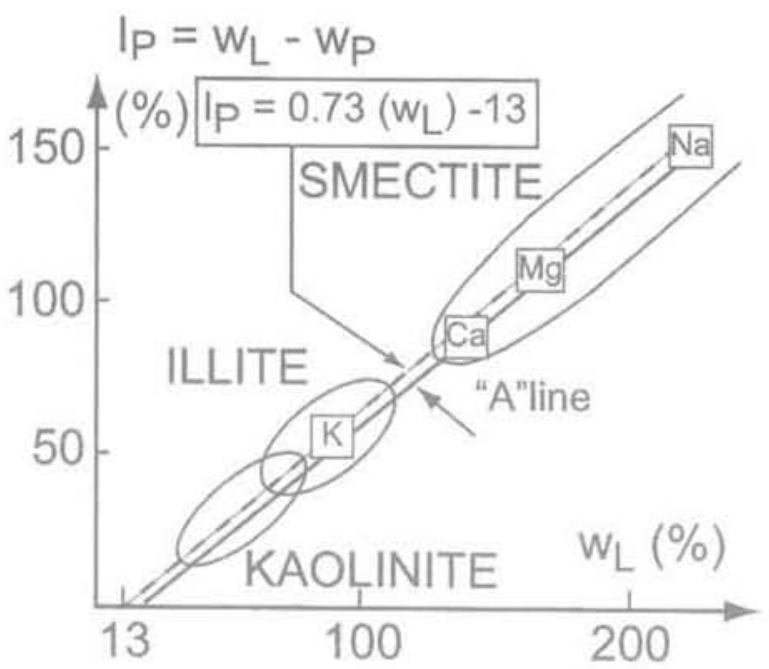

FiG.1 Abaque des argiles minérales. Mineral clay abacus.

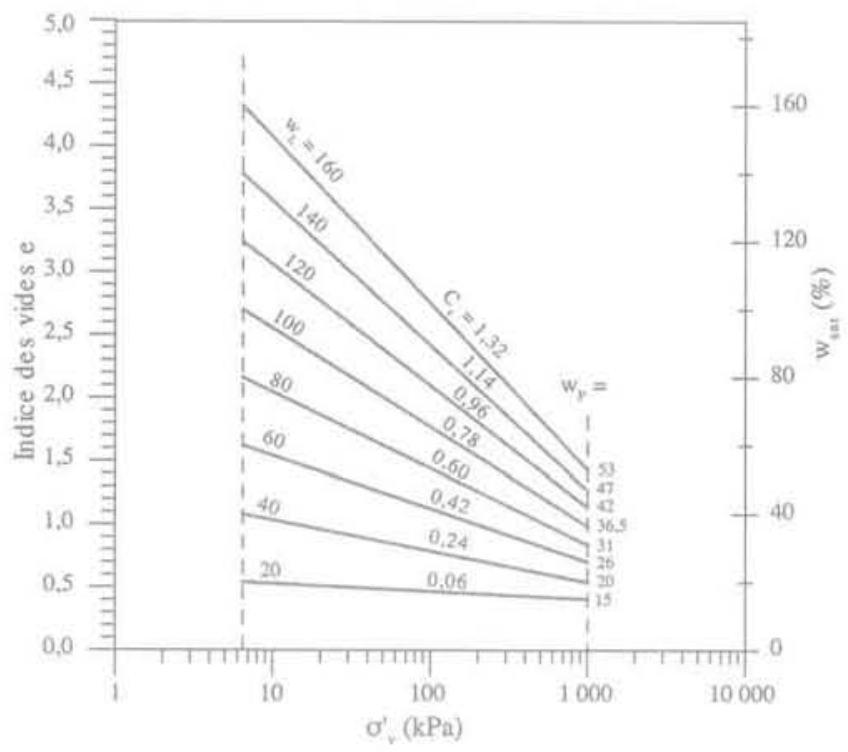

คG.2 Abaque de la consolidation œedométrique, Oedometric consolidation abacus.

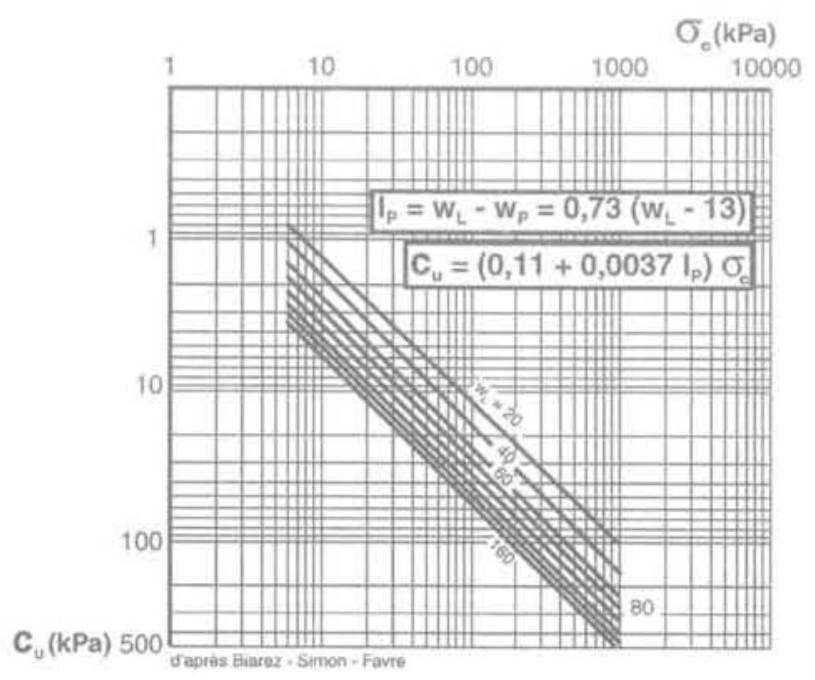

FIG. 3 Abaques des argiles remaniées. Remoulded clay abacus. 


\section{Nature et arrangement des grains de l'argile GoG}

\section{1}

\section{L'argile GoG (golfe de Guinée)}

Une carotte STACOR de $17 \mathrm{~m}$ de long a été prélevée par $700 \mathrm{~m}$ de fond sur le site B au large du golfe de Guinée. Elle a été fournie, débitée en tronçons de $1 \mathrm{~m}$ numérotés en fonction de leur profondeur, et stockés verticalement dans une chambre froide. Ainsi le tronçon se trouvant par exemple entre $7,10 \mathrm{~m}$ et $8,10 \mathrm{~m}$ de profondeur sera numéroté $7-8$. Chaque tronçon a été débité en 7 sous-tronçons (numérotés X 7-8, X variant de 1 à 7 , Fig, 4a), pour effectuer un maximum de mesures suivant un pas régulier pour analyses statistiques et géostatistiques. Le matériau apparait comme une argile très plastique de couleur gris foncé, saturée et comportant de nombreux débris coquillés. Ceux-ci sont visibles à l'cil nu, comme le montre la figure $4 \mathrm{~b}$ de l'argile à l'état sec, leur distribution causant une certaine hétérogénéité.

\section{2}

\section{Nature des " grains "}

Deux évolutions de $w_{L}$ et $w_{p}$ sont mises en évidence dans sur la figure 5. Globalement, les deux limites, variant de 110 à $160 \%$ pour $w_{\text {, }}$ et de 30 à $90 \%$ pour $w_{p}$, décroissent en escalier jusquáa 7 à $8 \mathrm{~m}$ de profondeur, puis semblent rester constantes entre $7-8 \mathrm{~m}$ et $15 \mathrm{~m}$ (noté par l'intervalle $[7-8-15] \mathrm{m}$ ). Ces résultats indiqueraient deux parties dans le sol, [1-7-8] m et [7-8-15] m, qui se différencient par la nature de leurs « grains », ce terme que nous utiliserons souvent pouvant désigner également un agrégat formé d'un ensemble de particules argileuses avec des éléments siliceux et organiques. L'analyse du degré argileux du sol par le bleu de Méthylène, présentée parallèlement aux limites d'Atterberg dans la figure 5, montre une variation, là aussi en escalier du VBS croissante dans la tranche [1-7 8] m. Cette variation qui semble s'opposer à

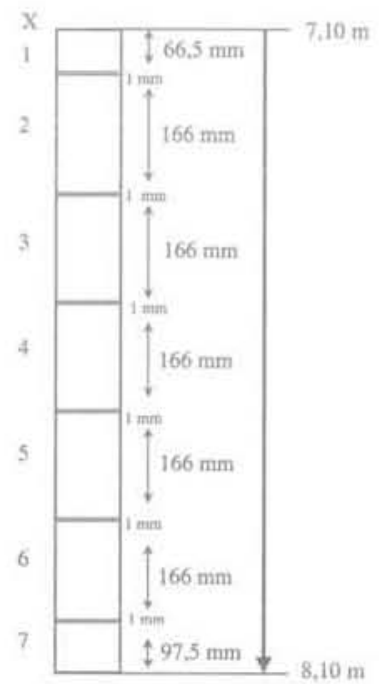

celle de $w_{L}$, viendrait de proportions argileuses de natures minéralogiques différentes, entre [1-3] m, [3-5] m puis [5$7 \sim 8 \mathrm{~m}$, la fraction argileuse étant la même à $3 \mathrm{~m}$ et à $9 \mathrm{~m}$ (Puech et al., 2005; Fig. 6) et éventuellement entre ces profondeurs. A partir de $8 \mathrm{~m}$ la valeur du VBS se stabilise (comme pour les limites) autour de 4,5, la nature minéralogique ainsi que la proportion de la fraction argileuse restent donc constantes. La marche d'escalier entre 5 et $7 \sim 8 \mathrm{~m}$ est toutefois plus forte, dans les trois diagrammes particulièrement pour $w_{1}$ (autour de $110 \%$ ) et pour le VBS (autour de 3,4$)$, les points représentant $w_{p}$ étant plus dispersés. Cette dispersion se transmet sur les valeurs de l'indice de plasticité $\mathrm{I}_{\mathrm{p} .}$. Ainsi, sur l'abaque $\mathrm{I}_{\mathrm{p}}-\mathrm{w}_{\mathrm{L}}$ présenté en figure 7 , les points $-7 \mathrm{~m}$ (moins de $7 \mathrm{~m}$ ) et $+7 \mathrm{~m}$ (plus de $7 \mathrm{~m}$ ) correspondent à des argiles de type smectite. Quelques points à $I_{p}$ faible, à des profondeurs variant de 4 à $5 \mathrm{~m}$ de 8 à $9 \mathrm{~m}$ et vers $13 \mathrm{~m}$, témoignent, d'une légère présence d'éléments organiques qui perturbent les évolutions de la cohésion non drainée (Favre et Hattab, 2005).

\section{3}

\section{Arrangement des " grains"}

L'arrangement des grains et son évolution sont indiqués par la variation de la teneur en eau $\mathrm{w}_{\text {nat }}$ ou celle de l'indice des vides e, ainsi que la contrainte effective axiale $\sigma^{\prime}$, celle-ci ayant été calculée à partir du poids spécifique déjaugé $\gamma$ moyen par mètre pour les couches $-7 \mathrm{~m}$, et par $50 \mathrm{~cm}$ pour les couches $+7 \mathrm{~m}$. Le poids spécifique des grains solides, et de l'eau de mer sont $\gamma_{5}=26,5 \mathrm{kN} / \mathrm{m}^{3}$ et $\gamma_{w}=10,2 \mathrm{kN} / \mathrm{m}^{3}$.

Nous pouvons noter les fortes valeurs de la teneur en eau naturelle (Fig. 8), particulièrement en surface entre 1 et $5 \mathrm{~m}$, avec des valeurs allant jusqu'à $180 \%$. A partir de $7 \mathrm{~m}$ de profondeur, $\mathrm{w}_{\text {nnt }}$ semble se stabiliser autour de $120 \%$ et même augmenter (ce phénomène sera expliqué par l'analyse de la cohésion non drainée). Par ailleurs, la particularité observée en marche d'escalier dans les diagrammes des limites, semble se répéter dans ceux de $w_{n \text { at }} \sigma^{\prime}$ ne varie pas linéairement en fonction de la profondeur (Fig. 8a) puisque $w_{\text {nit }}$ (donc $\gamma^{\prime}$ ) n'est pas constant. Néanmoins, par effet d'intégration (lissage) la déviation par rapport à la linéarité est très faible, elle sera néanmoins significative pour la comparaison avec la pression de consolidation et le calcul de l'OCR.

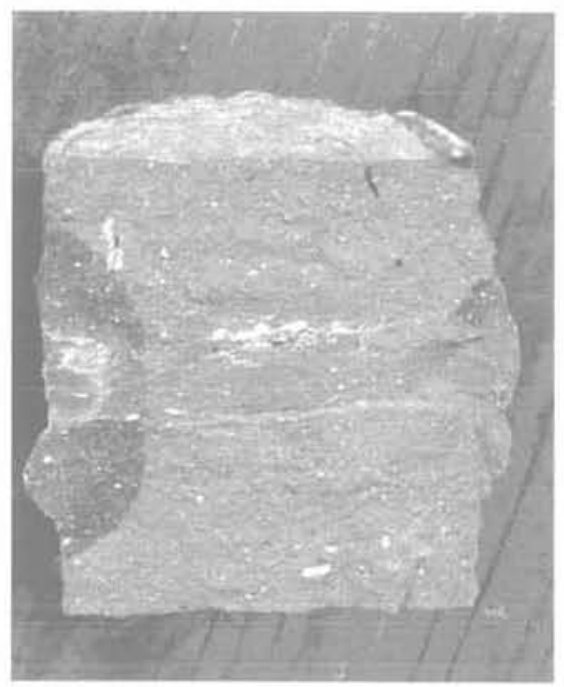

FG.4 Argile GoG. a) Carotte Tacore ; b) échantillon d'argile GoG à l'état sec. GoG clay. a) Stacore core cutting ; b) GoG sample. 
$\mathrm{w}_{p}(\%)$

$\begin{array}{lllllll}0 & 20 & 40 & 60 & 80 & 100 & 120\end{array}$

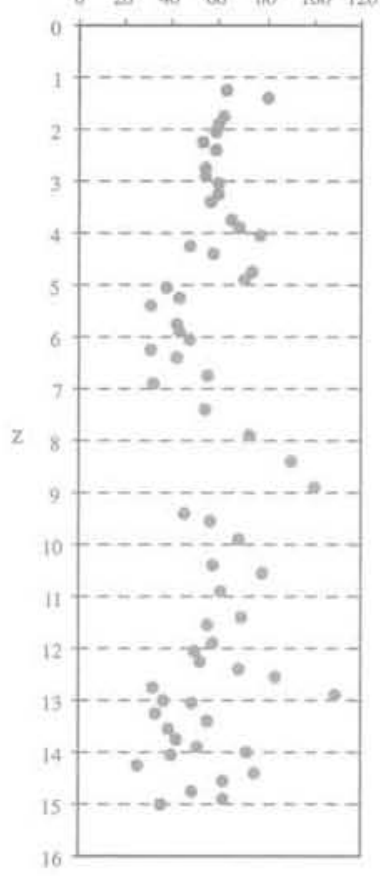

$w_{1}(\%)$

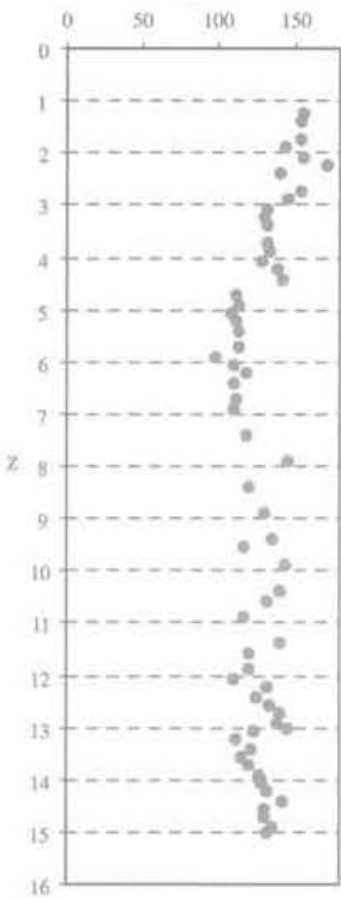

VBS

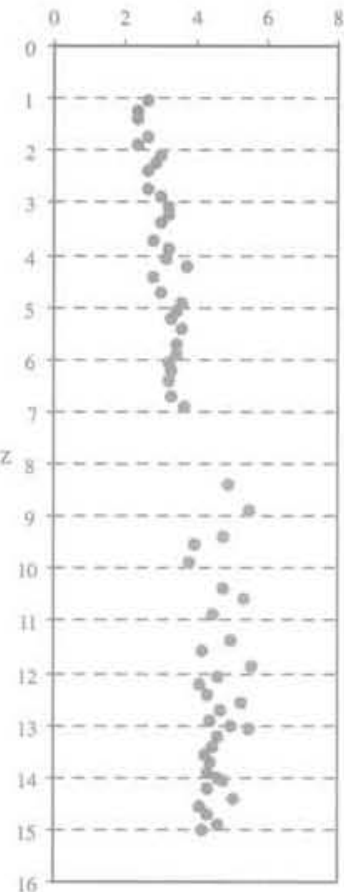

FG.5 Variation des limites $w_{L}$ et $w_{p}$ et du VBS en fonction de la profondeur. Variation of limits $w_{1}$ and $w_{y}$ and of MBV in function of depth.

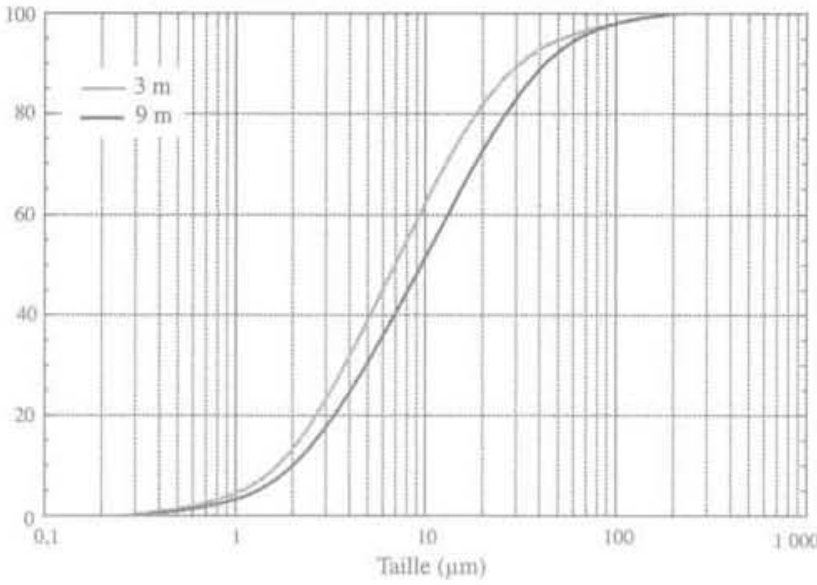

FiG.6 Courbe granulométrique à $3 \mathrm{~m}$ et à $9 \mathrm{~m}$ de profondeur.

Grain size distribution after Puech et al. (2005).

Dans les corrélations proposées précédemment, les argiles minérales, remaniées, normalement consolidées doivent se situer autour de l'équation 7, représentée par la droite NCRS (courbe normalement consolidée remaniée simplifiée) de la figure 9 ; les mêmes argiles surconsolidées, se situent en dessous de cette droite. La variation $I_{L}=\frac{W-W_{p}}{W_{L}-W_{p}}$ en fonction de $\sigma_{v^{\prime}}^{\prime}$ calculée pour l'argile GoG, donne un nuage de points au-dessus de NCRS (Fig. 9). Cette observation met en évidence une structure avec un arrangement particulier de ( grains »liés entre eux, ce qui les empêche de se resserrer « normalement $»$ comme cela pourrait être le cas pour une argile remaniée reconstituée au laboratoire. Ce liant pourrait être une légère cohésion de type physico-chimique, que nous mesurons plus loin au Fall Cone et au scissomètre de laboratoire, et par ailleurs

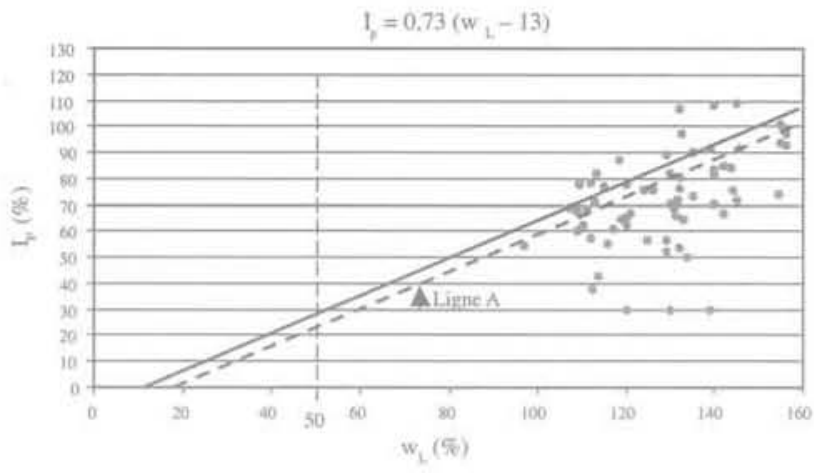

FG.7 Résultats expérimentaux comparés à l'abaque $\mathrm{I}_{\mathrm{p}}-\mathrm{w}_{1}$.

Experimental results in comparison with $l_{7}-W_{t}+$

obtenue par De Gennaro et al. (2005) dans l'argile GoG du site A. Le plan ( $\left.\sigma^{\prime}\right)$ (Eig. 10) permet de représenter ces résultats au vu de la courbe ICL (intrinsic compression line) et la SC.L (sedimentation compression line) proposées par Burland (1990). I. est l'indice des vides normalisé, équivalent d'une certaine manière à $\mathrm{I}_{\mathrm{L}}$, et défini par $I_{v}=\frac{e-e_{100}^{*}}{e_{100}^{*}-e_{1000}^{*}} \cdot e^{e_{100}}$ et $e^{*}{ }_{1000}$ sont les valeurs de l'indice des vides, pouvant être obtenues sur chemin cdométrique, pour respectivement $\sigma_{\mathrm{y}}^{\prime}=100 \mathrm{kPa}$ et $\sigma_{\mathrm{y}}^{\prime}=$ $1000 \mathrm{kPa}$, et corrélées aux limites $w_{\mathrm{L}}\left(\right.$ ou $e_{\mathrm{L}}$ ) et $\mathrm{w}_{\mathrm{p}}$ (ou $\mathrm{e}_{\mathrm{p}}$ ) (corrélation 10).

$$
\left\{\begin{array}{l}
\mathrm{e}^{*}{ }_{100}=0,109+0,679 \mathrm{e}_{\mathrm{L}}-0,089 \mathrm{e}_{\mathrm{L}}^{2}+0,016 \mathrm{e}_{\mathrm{L}}^{3} \\
\mathrm{C}^{*}{ }_{\mathrm{c}}=\mathrm{e}^{*}{ }_{100}-\mathrm{e}^{*}{ }_{1000}=0,256 \mathrm{e}_{\mathrm{L}}-0,04
\end{array}\right.
$$

La ICL (en pointillés) représente la compressibilité des argiles remaniées, telle que pour $\mathrm{e}=\mathrm{e}^{*}{ }_{100}$ et pour $\mathrm{e}=\mathrm{e}^{*}{ }_{100 \%} \mathrm{I}_{\mathrm{v}}=-1$. Son équation est exprimée par (11):

$$
L_{v}=2,45-1,285 \log \sigma_{v}^{\prime}+0,015\left(\log \log \sigma_{v}^{\prime}\right) 3
$$



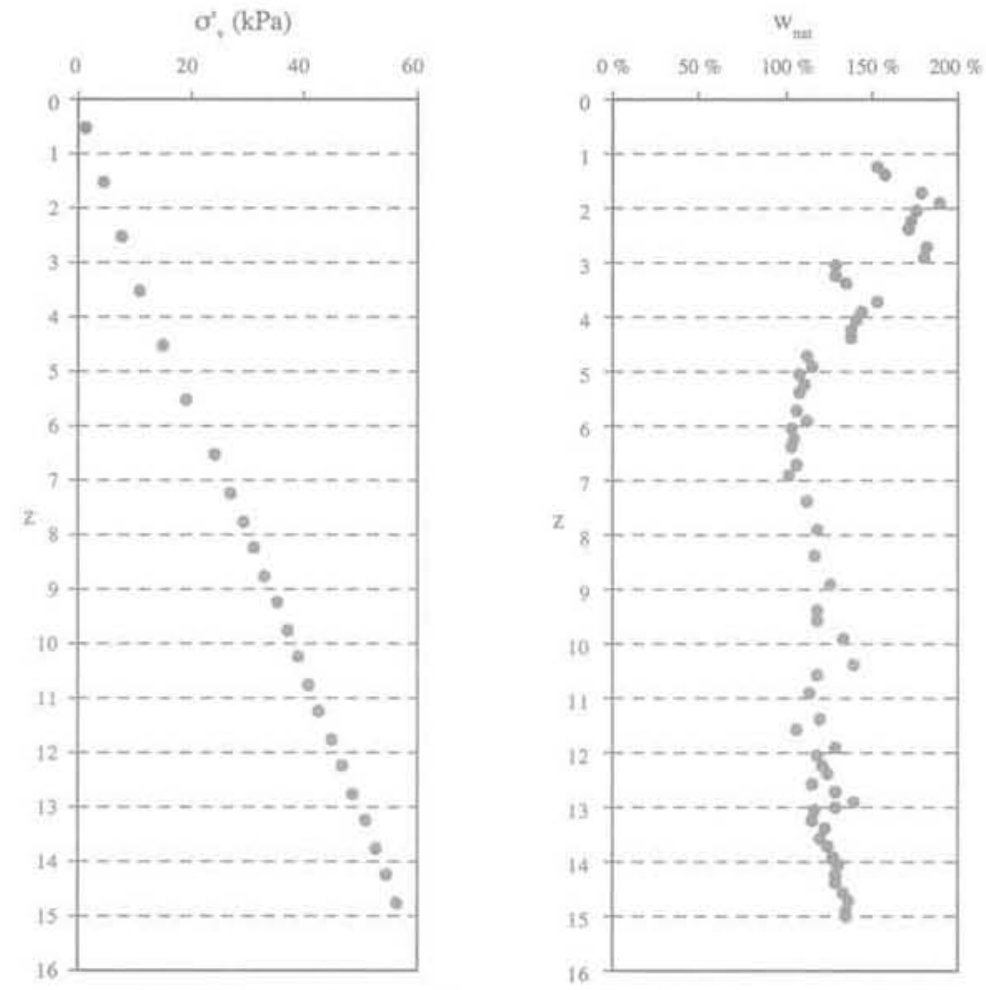

FlG. 8 Paramètres « d'arrangement des grains $»$ en fonction de la profondeur.

Parameters of $\alpha$ arrangement of grains $n$ in function of depth.

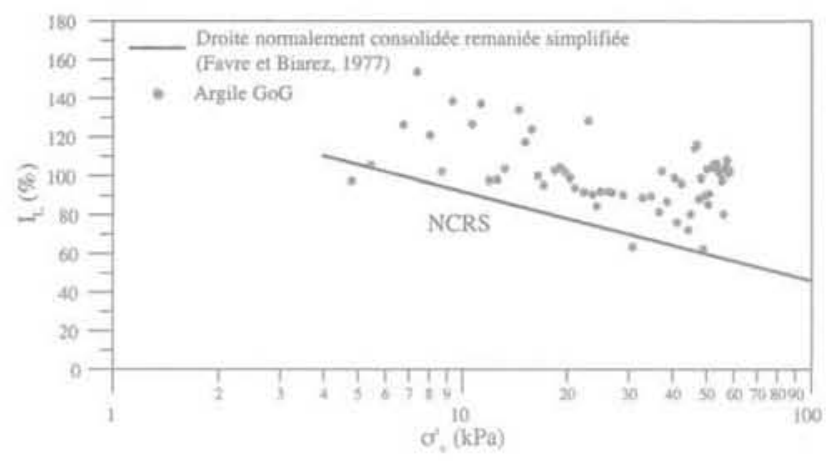

FIG:9 Variation $\mathrm{I}_{1}-\sigma^{\prime}$. $\left(\mathrm{I}_{\mathrm{L}}-\sigma_{v}{ }^{\prime}\right)$ variation.

La SCL (en trait plein) lui est approximativement parallèle. Celle-ci représente la compressibilité des argiles naturelles, elle a été obtenue à partir des résultats sur un grand nombre d'argiles sédimentées, pour la plupart en fond marins, collectées par Skempton (1970). Les points expérimentaux ont été définis en prenant l'état initial in situ et la limite de liquidité à chaque profondeur pour calculer $\mathrm{e}^{*}{ }_{100}$ et $\mathrm{e}^{\star}{ }_{1000}$ d'après (10) et (11). Les résultats sur le plan $\left(\mathrm{I}_{\mathrm{v}} \sigma^{\prime}\right)$, montrent des points principalement concentrés au-dessus mais très proches de la SCL, un domaine de sédimentation autour de la SCL semble ainsi se distinguer (Cotecchia et Chandler, 1997).

\section{4}

\section{Déformabilité}

\section{sur chemin œdométrique}

Les essais œdométriques sont réalisés en chargement-déchargement sur des échantillons, pris dans les

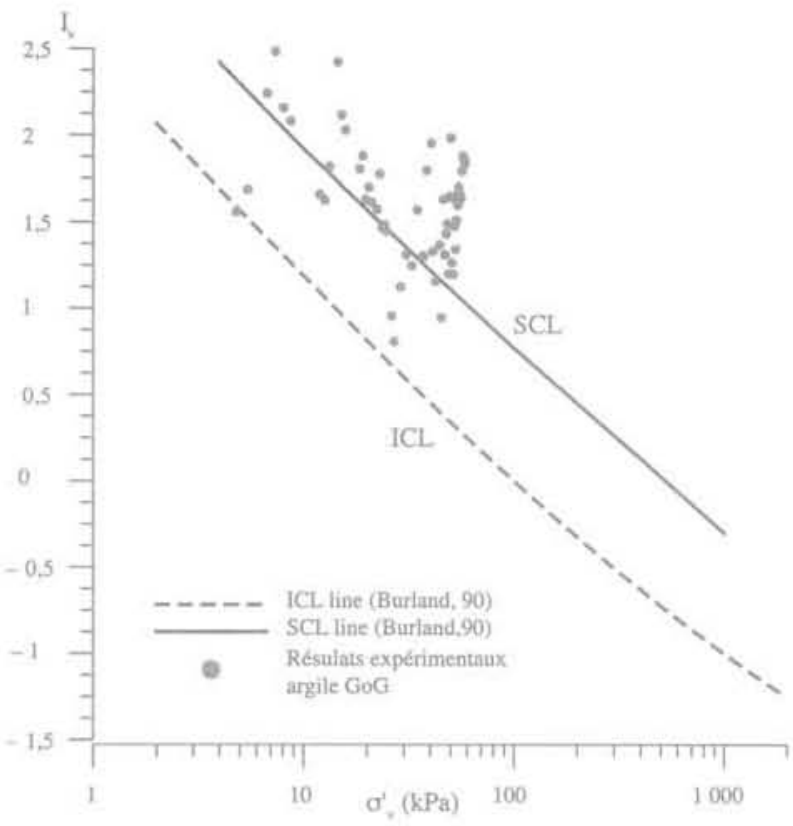

FG. 10 Résultats expérimentaux dans le plan (I $s^{\prime}$ ).

Experimental results in ( $\left.I_{v} s^{\prime}\right)$ plane.

sous-tronçons 2 et 5 , de hauteur $\mathrm{H}=24 \mathrm{~mm}$ et de diamètre $\mathrm{D}=70 \mathrm{~mm}$. Ces chargements sont effectués par paliers à des contraintes effectives allant jusqu'à $80 \mathrm{kPa}$ pour les couches $-7 \mathrm{~m}$ de profondeur, et à plus fortes contraintes, jusqu'à $250 \mathrm{kPa}$ pour les couches $+7 \mathrm{~m}$. Un essai sur l'échantillon (2 16-17) à 16,25 m de profondeur s'est, quant à lui, poursuivi jusqu'à une contrainte de $1050 \mathrm{kPa}$, soit 17 fois la contrainte effective in situ $\sigma_{\mathrm{v}}^{\prime}=$ $61 \mathrm{kPa}$, ce afin de suivre le comportement jusqu'aux fortes contraintes. Au vu de la courbe (2 16-17), la 
figure 11 montre les résultats pour les profondeurs $-7 \mathrm{~m}$, et la figure 12 pour les profondeurs $+7 \mathrm{~m}$. Sur ces plans, les échantillons sont indiqués en fonction des cotes du tronçon et par la nature du sous-tronçon (ST), les traits pleins correspondant au sous-troncon 2 et les traits discontinus aux sous-tronçons 5 . Toutes les caractéristiques mécaniques déduites à partir de ces résultats sont ensuite représentées en fonction de z.

La figure 13 donne deux tendances de $\alpha$ surconsolidation , le sol parait normalement consolidé entre 8 et $15 \mathrm{~m}$ avec des OCR variant autour de 1, et légèrement surconsolidé entre 2 et $8 \mathrm{~m}$, avec des OCR variant entre
1 et 1,7 ; toutefois, nous avons à $5 \sim 6 \mathrm{~m}$ un point particulier pour lequel $\mathrm{OCR}=0,7$, La figure 14 montre que l'indice de compression $\mathrm{C}_{\vec{c}}$, variant en moyenne autour de 1,5 sur toute la profondeur, est quasi systématiquement supérieur à la corrélation (4) : mis à part quelques points entre 2 et $6 \mathrm{~m}$, dont les essais effectués à faible chargement ont sous-estimé les valeurs de $\mathrm{C}_{\mathrm{c}}$.

Ce décalage montre que l'influence de facteurs rattachés aux conditions de dépôt et aux événements qui le suivent (lessivage) (Burland, 1990) devient, pour cette argile, significative ; et cette compressibilité plus forte vient, comme cela est schématisé en figure 15 , de la

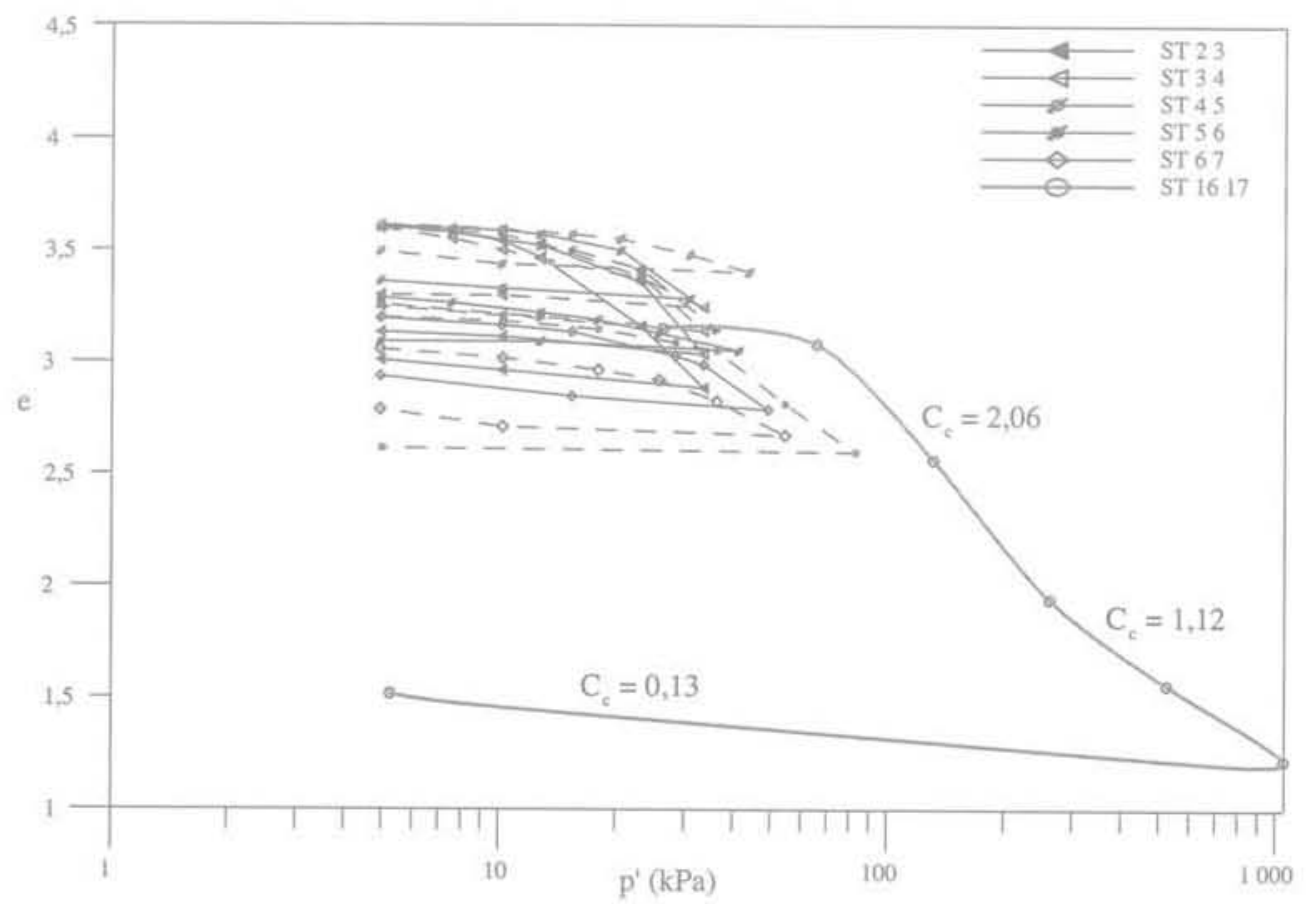

FIG.11 Résultats œdométriques par $-7 \mathrm{~m}$. Oedometric results at $-7 \mathrm{~m}$.

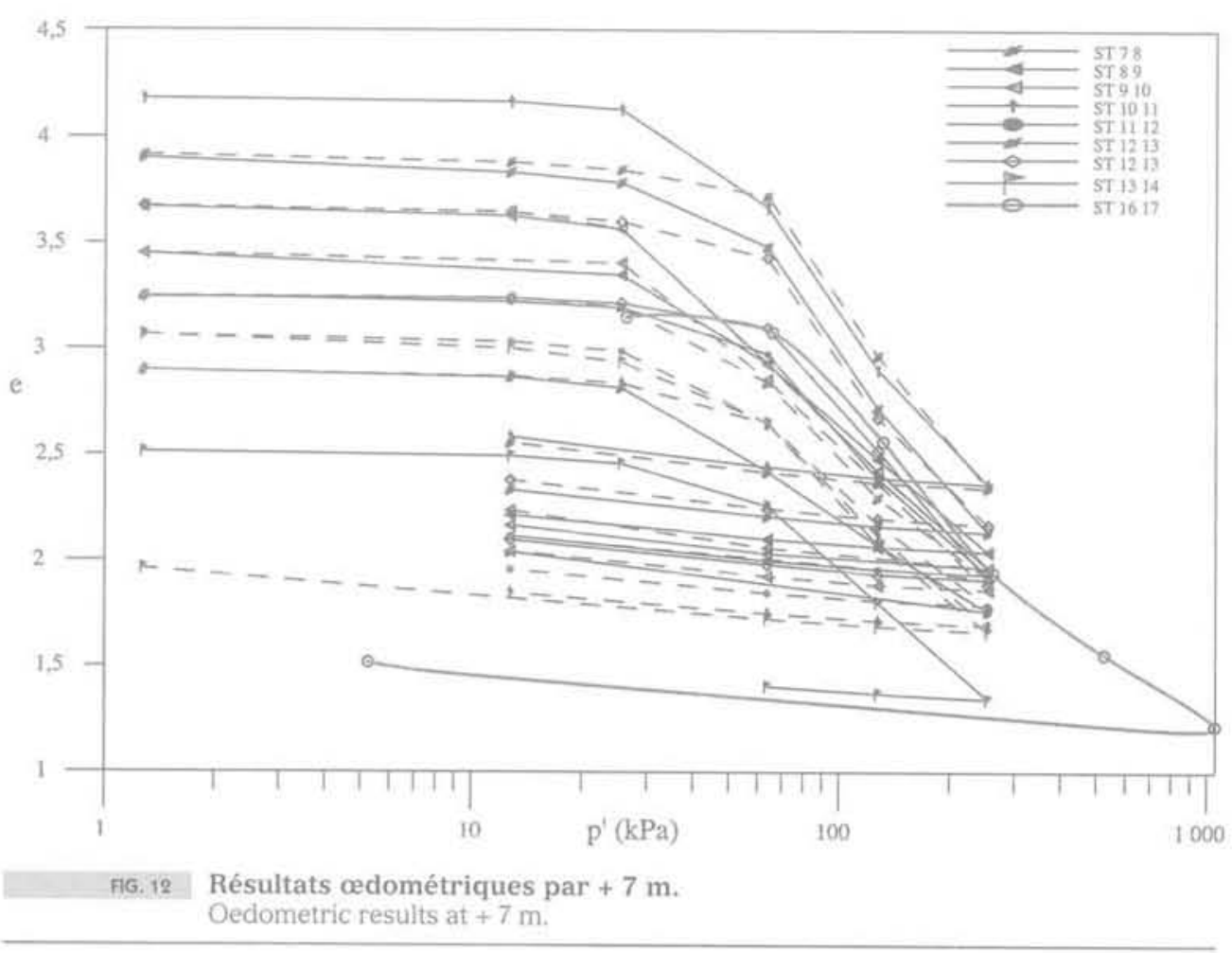



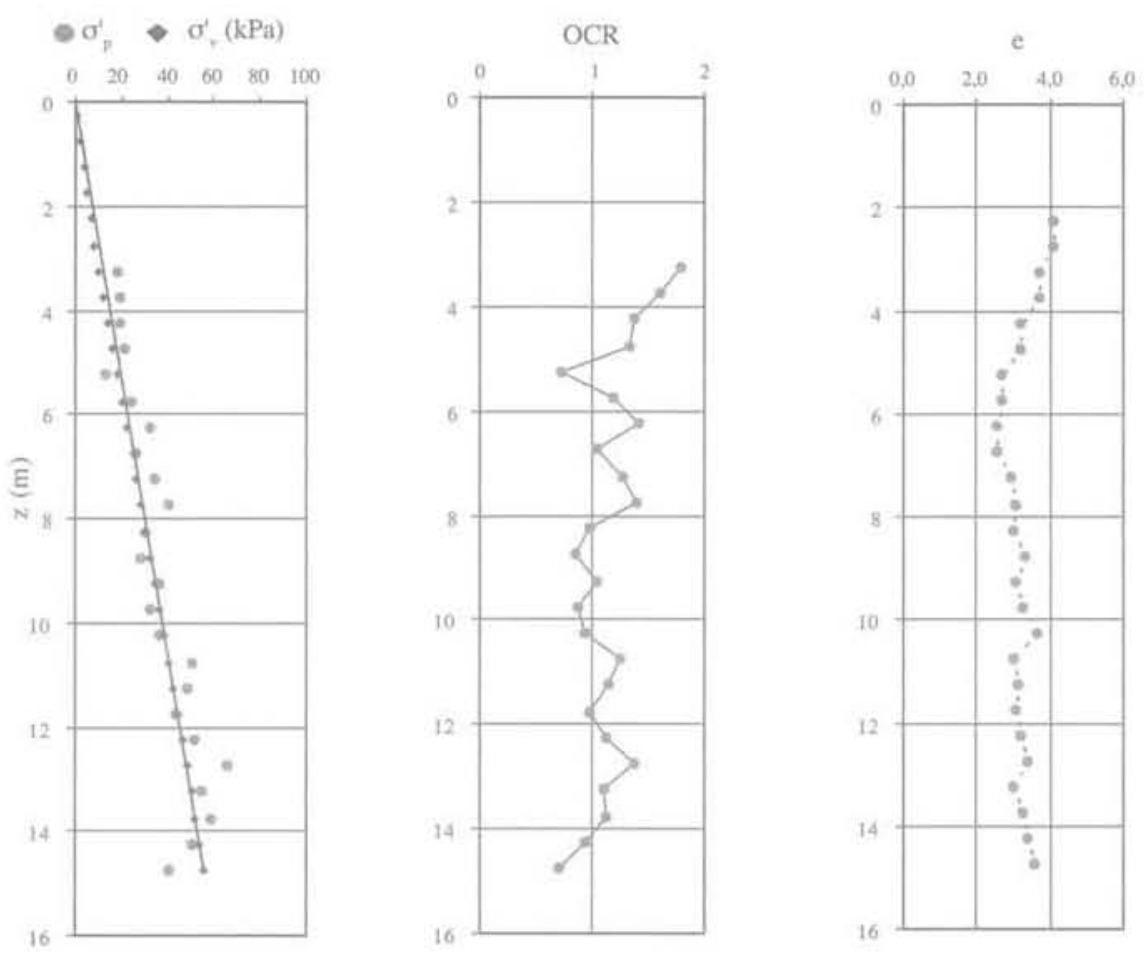

FG. 13 Variation de $\sigma_{v}^{\prime}, \sigma_{p}^{\prime}$, OCR et e (in situ) en fonction de la profondeur. $\sigma_{,}^{\prime}, \sigma_{t}^{*}, \mathrm{OCR}$ and $\mathrm{e}(\mathrm{in}$ situ) in function of the depth.

C

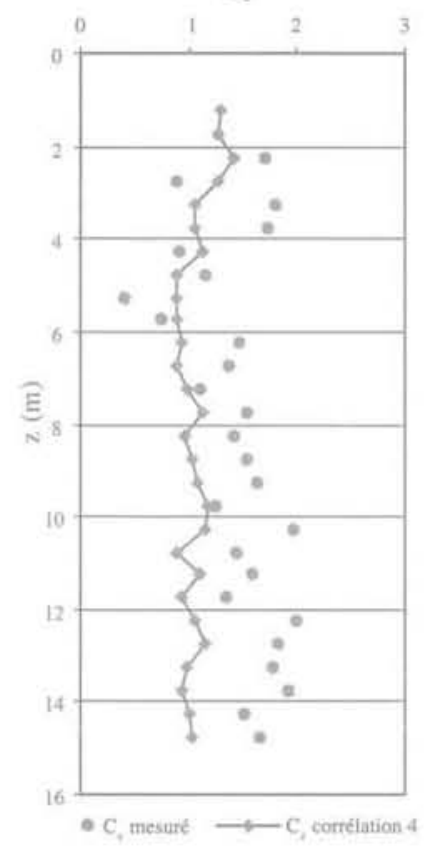

C

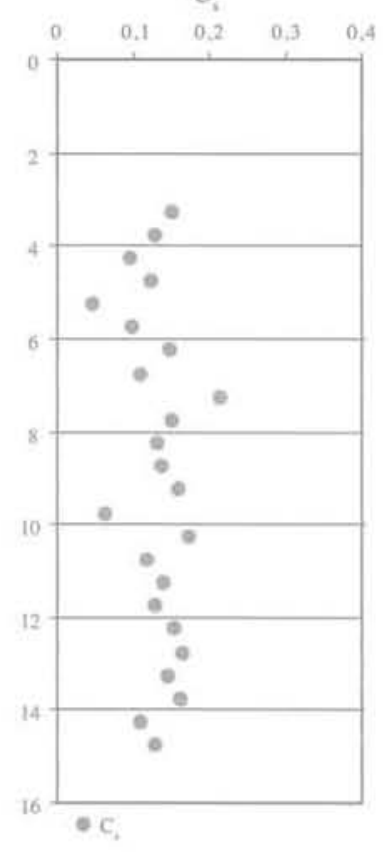

$\mathrm{C}_{\mathrm{f}} / \mathrm{C}_{\text {. }}$

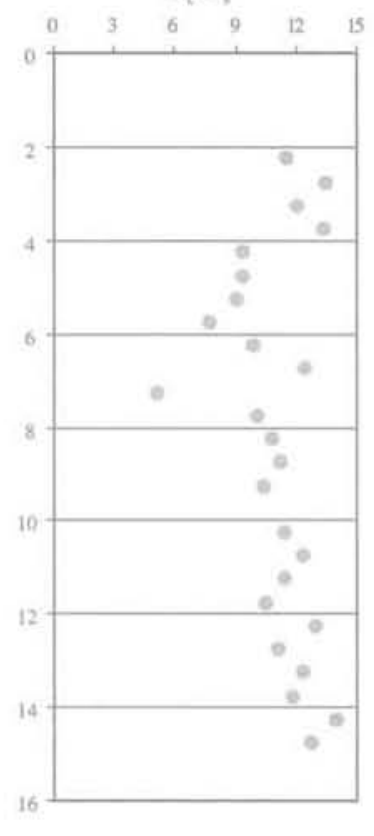

FIG.14 Variation de $\mathrm{C}_{f}$ et $\mathrm{C}_{\text {s }}$ en fonction de la profondeur. $\mathrm{C}_{\varepsilon}$ and $\mathrm{C}_{s}$ in function of the depth.

destruction progressive des liens entre " grains » lors du chargement œdométrique. Lorsque, à forte contrainte, tous les liens ont été détruits, le comportement rejoint celui de l'argile remaniée (Biarez et Hicher, 1994 ; Burland, 1990). Les modules œedométriques $\mathrm{E}_{\text {oedo }}$ passent de $55 \mathrm{kPa}$ environ à $2 \mathrm{~m}$ à $330 \mathrm{kPa}$ à $15 \mathrm{~m}$ dans un rapport de 0,75 à 0,6 avec la référence. L'indice de gonflement $C_{5}$ semble stable autour de 0,13 également sur toute profondeur, par contre, le rapport $\mathrm{C}_{\mathrm{c}} / \mathrm{C}_{3}$ affiche des valeurs très fortes comparé à la corrélation (8), soit de 10 à 12 au lieu de 4 à 6 dans les argiles remaniées. Nous pouvons affirmer, pour l'argile GoG, que le comportement est de type normalement consolidé non remanié, le OCR obtenu au niveau du coude de la courbe œdométrique, met en évidence la cimentation. La seconde tendance de la courbe qui caractérise le 


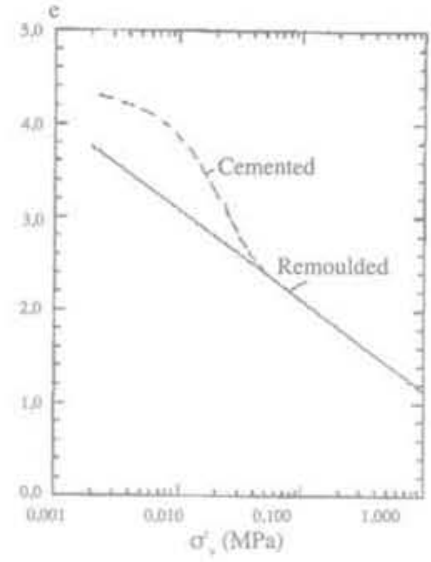

FIG, 15 Influence de la cimentation, d'après Biarez et Hicher (1994).

Influence of the cementation, after Biarez and Hicher (1994),

matériau lorsqu'il est remanié, correspond selon Burland (1990) à la courbe de compression intrinsèque ICL. L'essai œdométrique (ST 2 16-17) réalisé jusqu'à $1050 \mathrm{kPa}$ montre clairement cette évolution du comportement (Figs. 11 et 12). Deux indices de compression $C_{\text {c }}$ sont alors mis en évidence, l'un dans le domaine des faibles et moyennes contraintes $\left(\sigma^{\prime}\right.$ : $90 \mathrm{kPa} \approx 300 \mathrm{kPa}, \mathrm{C}_{f}=2,063$ ) et l'autre dans celui de fortes contraintes $\left(\sigma_{\mathrm{v}}^{\prime}>300 \mathrm{kPa}, \mathrm{C}_{\mathrm{c}}=1,115\right)$,

Un calcul de l'expression de la ICL à partir de (11) et (10) en fonction du paramètre $I_{\text {, }}$, montre une estimation plus forte de la compressibilité par rapport à NCRS, exprimée par la relation (7). La représentation
(Fig. 16) dans le plan $\left(I_{L} \sigma^{\prime}\right)$ montre qu'en effet la $I C L$ (en traits discontinus) se situant en dessous de NCRS (en trait continu), les deux courbes sont approximativement parallèles à partir de $\sigma_{v}^{\prime}=100 \mathrm{kPa}$. Les chemins œdométriques, reportés également dans le plan normalisé (I, $\sigma^{\prime}$ ) de la figure 16, évoluent vers un coude localisé d'une manière général au-dessus de NCRS. Un domaine caractérisant la sédimentation des sédiments GoG semble ainsi mis en évidence, comme cela a été constaté plus haut. Par ailleurs, il n'apparait pas de distinction particulière entre les courbes par $-7 \mathrm{~m}$ de profondeur (en trait continu) et $+7 \mathrm{~m}$ (en traits discontinus), mis à part le fait de ces derniers vont plus loin en chargement. Alors que NCRS semble représenter une asymptote pour le chemin cedométrique sur de la kaolinite P300 reportée dans ce plan, les courbes de l'argile GoG subissent une plus forte compressilibité et semblent aller rejoindre la ICL, ceci est particulièrement évident dans le cas de ST 16-17. L'hétérogénéité de l'argile GoG notamment avec un grand nombre de débris coquillés qui continueraient à s'écraser, ainsi que la compressibilité des " grains » eux-mêmes, peuvent expliquer cette différence avec les argiles minérales plus homogènes de laboratoire.

\section{5}

\section{Rupture en non drainé}

La cohésion non drainée (Fig. 17) a été mesurée au Fall Cone $\left(\mathrm{c}_{\mathrm{uf}}\right)$ figurée par des carrés et au scissomètre de laboratoire $\left(\mathrm{c}_{\mathrm{u}}\right.$ ) figurée par des ronds. Leurs variations sont comparées à l'équation de Skempton (5) ( $\mathrm{c}_{\mathrm{v}}$ ), figurée par des losanges, pour les argiles minérales remaniées normalement consolidées. Ces résultats

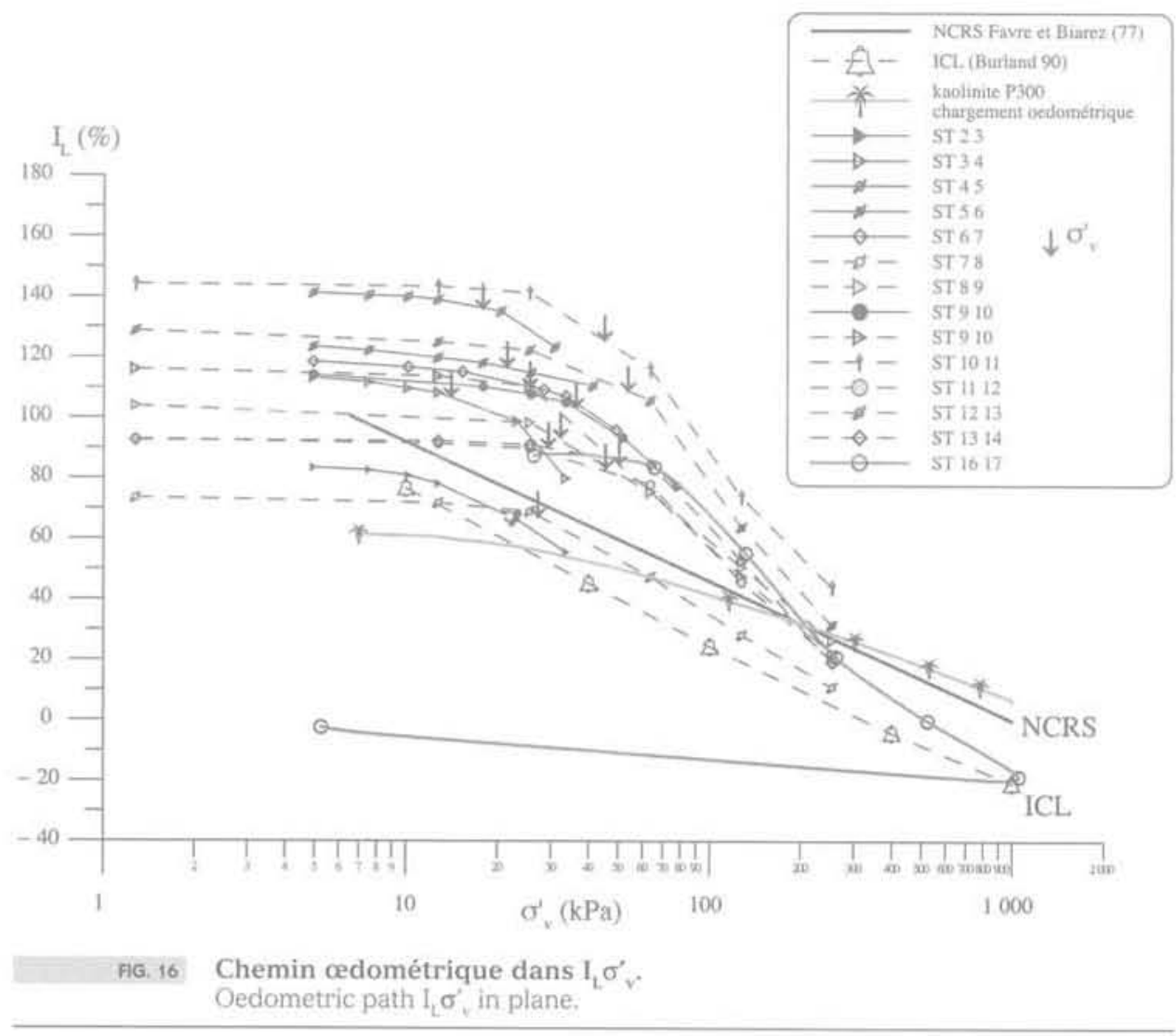


c.

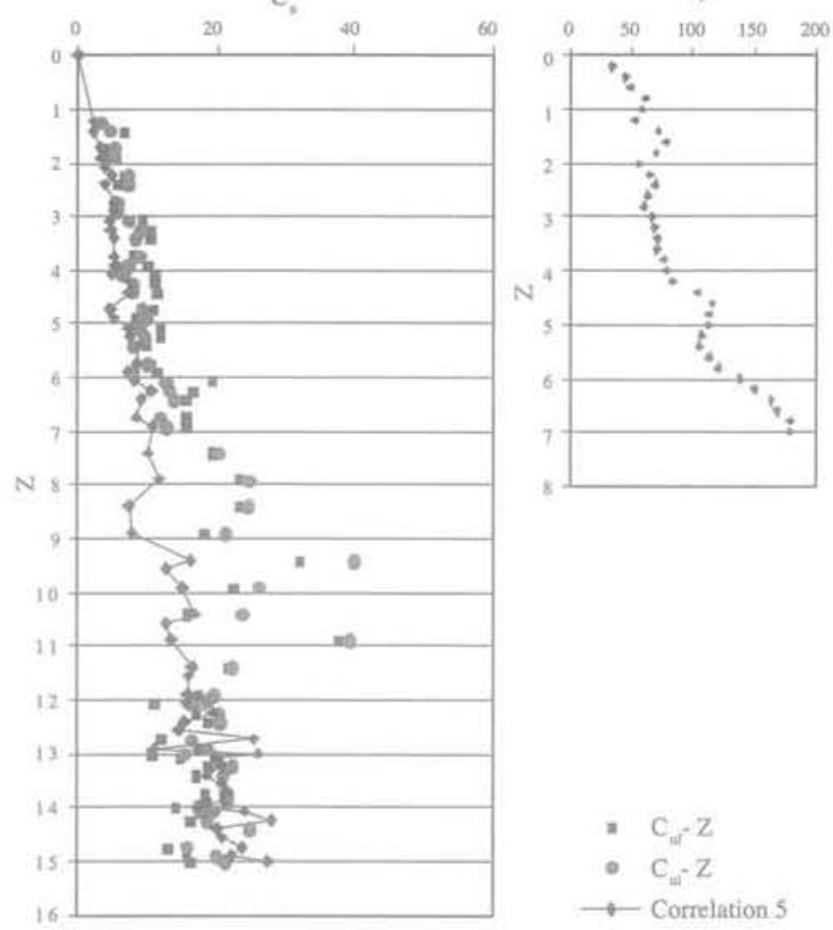

AG. 17 Profil des cohésions non drainées $\left(\mathrm{c}_{\mathrm{us}}, \mathrm{c}_{\mathrm{ur}}\right.$ $\left.c_{u}\right)$, et profil pénétrométrique:

Undrained cohesion profiles $\left(\mathrm{c}_{\mathrm{ur}}, \mathrm{c}_{\mathrm{ur}}, \mathrm{c}_{\mathrm{u}}\right)$, and penetrometric profile $\mathrm{q}_{\mathrm{c}}$

mettent en évidence un $\mathrm{c}_{\mathrm{uf}}$ quasi systématiquement supérieur au $c_{w}$ sur $-7 \mathrm{~m}$ et inférieur sur $+7 \mathrm{~m}$. Ainsi, une réponse non indépendante des appareils pour deux sols différents serait possible. D'autre part, les mesures sont légèrement supérieures à l'équation (5) de Skempton de 1 à 7 mètres environ mettant en évidence la cohésion naturelle de type physico-chimique, déjà constatée auparavant. En revanche, les mesures entre 7 et 9 mètres s'éloignent fortement de la corrélation pour s'en approcher à partir de $9 \mathrm{~m}$ et la rejoindre vers 12 13 mètres. Elles restent cohérentes entre Fall Cone et scissomètre de laboratoire. Ce type d'évolution en fonction de la profondeur pourrait s'expliquer par un phénomène de décapage-remblaiement, ces phénomènes de variations de $c_{\text {ur }}$, ayant également été constatées par Matsuo et al. (1975) sur les dépôts portuaires ayant subi les phases de remblaiement dans le port de Nagoya : la cohésion non drainée présentait une variation de type de la ligne $\mathrm{M}$ (Fig. 17). On aurait affaire à un dépôt précédent qui aurait été décapé de moins de 7 à 8 mètres (glissement marin), puis remblayé par un nouveau dépôt de 7 à 8 mètres. Cette hypothèse explique également la variation constatée dans le profil de $w_{\text {nat. }}$ Par ailleurs, les variations en escalier constatées entre $1 \mathrm{~m}$ et $7 \mathrm{~m}$, pour $\mathrm{W}_{\mathrm{t}}, \mathrm{W}_{\mathrm{p}}$, VBS et $\mathrm{W}_{\mathrm{rur}}$ ont été confirmées, par une analyse en composantes principales (ACP) sur le tableau transposé (Favre et al., 2004), de la cohésion non drainée et des paramètres de nature et d'arrangement des grains. Elles pourraient conduire à distinguer 3 sous-couches dans les $-7 \mathrm{~m}$; de 1 à 2 mètres, de 2 à 5 mètres et de 5 à $7-8$ mètres (Fig. 18). Les variations de la résistance de pointe pénétrométrique sur $-7 \mathrm{~m}$ (Fig. 17), mesurées à une distance voisine du site $B$ d'une dizaine de mètres, évoluent également dans le même sens donnant un $\mathrm{N}_{k}=8,4$ dans le cas du Fall Cone et un $N_{k}=11,1$ dans le cas du scissomètre de laboratoire.

\section{6}

\section{Conclusion}

Une analyse extrêmement fine suivant un pas de 16,7 $\mathrm{cm}$, d'un grand nombre de variables tant physiques, $\mathrm{w}_{\mathrm{L}}$ $\mathrm{W}_{\mathrm{p}}, \mathrm{VBS}, \mathrm{w}_{\text {nat }}$ que mécaniques $\mathrm{C}_{\mathrm{c}^{\prime}} \mathrm{C}_{\mathrm{s}^{\prime}} \mathrm{OCR}, \mathrm{c}_{\mathrm{u}}$ fatl-cone, $\mathrm{c}_{\mathrm{u}}$ scisso-lab, $\mathrm{q}_{c}$, a été faite sur une carotte Stacor de très bonne qualité de $17 \mathrm{~m}$ prélevée par $700 \mathrm{~m}$ de profondeur dans le golfe de Guinée. Outre la modélisation géostatistique en cours de publication, de cette étude, un certain nombre de conclusions, sur la nature de l'argile marine grande profondeur GoG, peuvent être avancées. L'argile est de type smectite, avec des débris coquillés, des éléments organiques, siliceux, carbona-

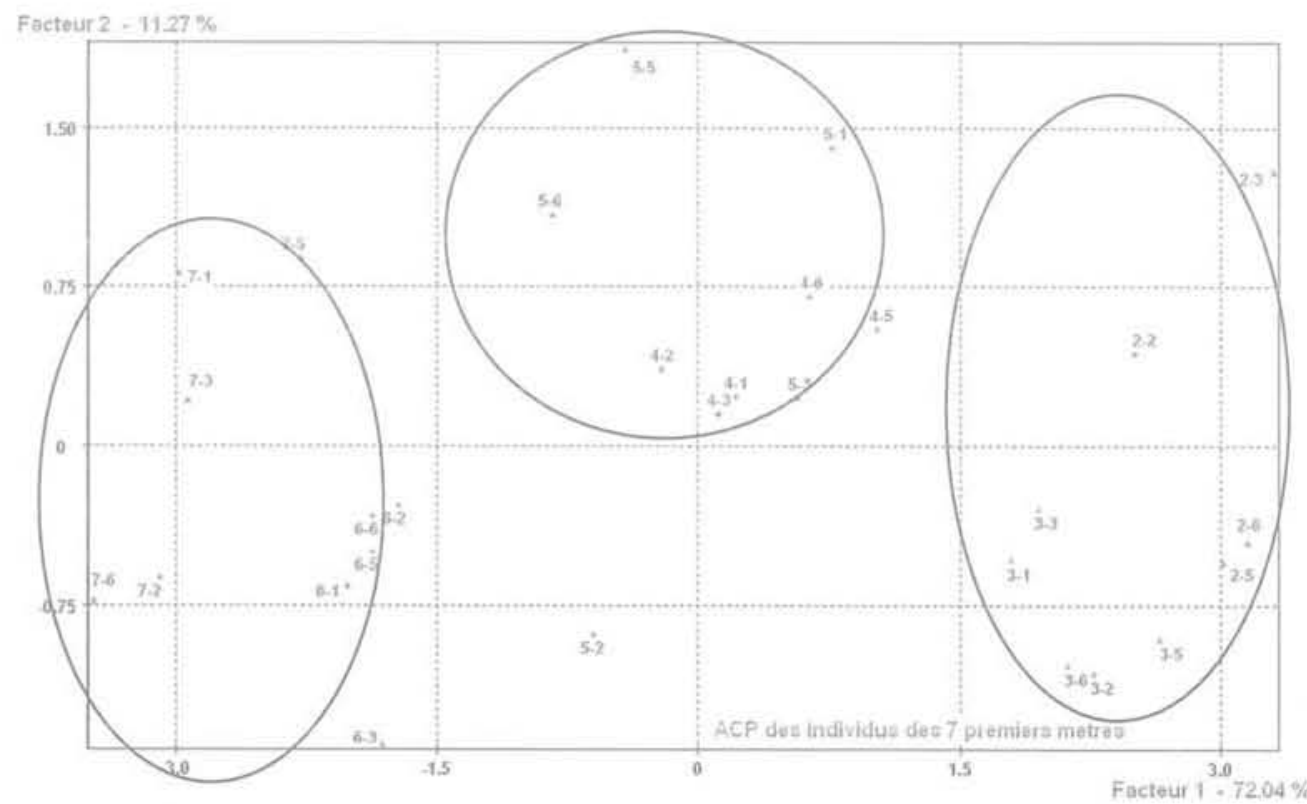

FIG. 18. Analyse en composantes principales (ACP). Principal components analysis (PCA). 
tés. Sa composition minéralogique apparaît comme riche, complexe et vraisemblablement non uniforme sur $17 \mathrm{~m}$ de profondeur. La confrontation de ses paramètres avec les comportements de référence, des argiles remaniées de laboratoire et avec l'abaque de sédimentation de Burland, permet de mettre en évidence un phénomène de sédimentation à fort indice des vides (abaque Burland). Celui-ci correspond à un auto-serrage des grains contrarié (abaque $I_{L}-\log \sigma_{\mathrm{v}}^{\prime}$ ) par une cohésion qui est une liaison de type physico-chimique. La cohésion non drainée s'en trouve augmentée de 1,2 à 1,3 par rapport à celle des argiles remaniées. Cette cohésion fait apparaitre une « surconsolidation » apparente dans les premiers mètres, qui ne dépasse pourtant pas ici 2, un coude prononcé (argile sensible) dans les chemins œedométriques donnant un rapport $C_{c} / C_{s}$ très supérieur à celui des argiles remaniées.
Enfin, le pas très fin de mesure a permis de mettre en évidence une genèse complexe du site, avec un phénomène de décapage de moins de 7 à $8 \mathrm{~m}$ environ dont l'origine pourrait être intéressante à trouver, car il pourrait se reproduire et être dangereux pour les ouvrages des grands fonds. Puis, un phénomène de remblaiement de 7 à $8 \mathrm{~m}$ ayant pu se faire en 3 phases de nature distinctes de 2 à $3 \mathrm{~m}$ mètres environ chacune et correspondant à des cycles d'érosion terrestre et de transport-sédimentation.

\section{REMERCIEMENTS}

Les auteurs remercient la société Fugro-France qui a mis à leur disposition les 17 tronçons de la carotte Stacor du site B, ainsi que le CLAROM dans le cadre duquel s'est faite cette étude et qui l'a financée, et plus spécialement MM. Meunier. Nauroy et Puech pour les échanges scientifiques avec eux.

\section{Bibliographie}

Biarez J., Favre J.L. - «Parameters filing and statistical analysis of data in soils mechanics 3, Proc. 2 Int. Conf. Appl. Stat. Prob., Aix-la-Chapelle, 1975, vol. 2 p. 249.

Biarez J. Favre J.L. - " Statistical estimation and extrapolation from observations ). Rapport à la session spec. $n^{\circ} 6$, C.R. du $9^{e}$ congrès Int. Méc. Sols Trav. Fond, Tokyo, 1977, vol. 3, p. 505.

Biarez J., Favre J.L., Rudelle M. - $\alpha$ Application de l'analyse des correspondances à l'étude des sédiments marins carbonatés $\mathrm{x}$. Proc. special session 6 . Topic 2, édition. Ecole centrale Paris, 1977.

Biarez J., Fayad T., Taillez S., Gomes Correia A., Flavigny E., Branque D. a Argiles et craies du tunnel sous la Manche : grains sans et avec colle n, $2^{\text {nd }}$ Int. Conf. on Hard Soil and Soft Rocks. Balkema, 1998, vol. 1, p. 437-445.

Biarez J., Hicher P.-Y. - a Elementary mechanics of soils behaviour. Saturated remoulded soils 11. Balkema A.A., 1994. Rotterdam/Brookfield.

Burland.J.B. - «On the compressibility and

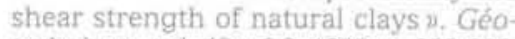
technique, vol. $40, \mathrm{n}^{\circ} 3,1990, \mathrm{p} .329-378$

Cotecchia F., Chandler R.J. - "The influence of structure on the pre-failure behaviour of a natural clayx. Géotechnique, vol. $47, n^{\circ} 3, \mathrm{p}, 523-544$.

Cozzolino E.V.M. - « Stastistical forecasting of compression index x. Proc. $5^{\text {th }}$ Int. Cont. Soils. Mech. Fond. Eng, Paris, 1961 , vol. 1, p. 51, Paris.
De Gennaro V., Puech A., Delage P. - «On the compressibility of deepwater sediments of the Gulf of Guinea ". Proc. Int. Symp. On frontiers in Offshore Geotechnics, Perth, Autralia, 2005.

Favre J.L. - «Table ronde sur les corrélations de paramètres en mécanique des sols ». Ss la dir. de Biarez, Ecole centrale Paris, 1972, vol, II, p. 1-51.

Favre J.L. - a Milieu continu et milieu ciscontinu. - Mesure statistique indirecte des paramètres rhéologiques et approche probabiliste de la sécurité $y$. Thèse de doctorat d'État ès sciences physiques, université Pierre-et-MarieCurie, Paris 6, 1980.

Favre J.L., Biarez J., Mekkaoui S. «Models for large strain behaviour of sands and remoulded clays in oedometer and triaxial tests $x$, PARAM 2002 International Symposium. LCPC/Presses de I'ENPCs Ponts, 2002, p. 369-385.

Favre J.L., Chabou D., Chauvet P. «Modélisation géostatistique des sols marins grande profondeur $\$$. Rapport de recherche, projet CLAROM, Ecole centrale Paris, 2005

Favre J.L., Hattab M. - « Propriétés des sédiments marins grande profondeury. Rapport de recherche, projet C.LAROM. Ecole Centrale centrale Paris. 2005.

Hattab M., Hicher P.Y. - " Dilating behaviour of overconsolidated clay $x$. Soils and Foundations, vol. 44, n ${ }^{\circ} 4,2004$. p. $27-40$.

Ismail M.A., Joer H.A., Randolph M.F., Meritt A. - «Cementation of porous materials using calcite w, Géotechnique, vol. $52, n^{\circ} 5,2002$, p. 313-324.

Lambe T.W., Martin M. - $\alpha$ Composition and Engineering Properties of soils D. Highway Research Board, vol. 36, 1957. p. 693-702.

Magnan J.P. - « Les méthodes statistiques et probabilistes en mécanique des sols m. Presses de l'ENPCs Ponts, Paris, 1982.

Matsuo M., Kuroda K., Asaoka A. *Uncertainties and decision in design of embankment. o Proc, $2^{\text {nd }}$ ICASP. Aachen sept. 1975, p. 143-145.

Puech A., Colliat J.L., Nauroy J.F., Meunier J. - uSome geotechnical specificities of Gulf of Guinea deepwater sediments n. Proc. Int. Symp. On Frontiers in Off'shore Geotechnics, Perth, Australia, 2005.

Ohtsubo M., Egashira K., Koumoto T., Bergado D.T. - $\alpha$ Mineralogy and chemistry, and their correlation with the geotechnical index properties of Bangkok Clay : Comparaison with Ariake Claym. Soils and Foundations, vol. 40, $\mathrm{n}^{\circ} 1,2000$, p. 11-21.

Skempton A.W. - « Les fondations (1954)», Léonards G.A. Dunod Paris, 1968.

Skempton A.W. - $\alpha$ Notes on compressibility of clays x. Q..I. Geol. Soc., 100, 1944, p. 119-135.

Skempton A.W - $\alpha$ The consolidation of clays by gravitational compaction ». $Q$. J. Geol. Soc. 125, 1970, p. 373-411.

Yin J.H. - "Properties and behaviour of Hong Kong marine deposits with different clay contents m. Canadian Geotechnical Journal, vol. 36, n* 6 p. 1085-1095. 